\title{
Regeneração natural em área de Caatinga no Baixo São Francisco sergipano: composição, diversidade, similaridade florística de espécies florestais
}

\author{
Natalie da Mota Soares ${ }^{1}$ Robério Anastácio Ferreira ${ }^{1}$ Higor dos Santos Vieira ${ }^{1}$ Janisson Batista de Jesus ${ }^{2}$ Diogo \\ Gallo de Oliveira ${ }^{1}$ Ana Cecília da Cruz Silva ${ }^{1}$
} ${ }^{1}$ Universidade Federal de Sergipe, Av. Marechal Rondon, s/n - Jardim Rosa Elze, São Cristóvão - SE, 49100-000
${ }^{2}$ Universidade Federal do Rio Grande do Sul, Av. Bento Gonçalves, 9500 - Centro Estadual de Pesquisas em Sensoriamento Remoto e Meteorologia
- Campus Vale, Porto Alegre - RS, 91501-970

*Author for correspondence: janisson.eng @ gmail.com

Received: November 2018 / Accepted: September 2019/ Published: September 2019

\section{Resumo}

O conhecimento da regeneração natural na Catinga é essencial para compreender processos ecológicos importantes que aí ocorrem, especialmente a sua dinâmica, levando-se em consideração a condição climática a qual está associada. Este trabalho foi realizado com o objetivo de compreender a regeneração natural, investigando-se a capacidade de resiliência de três ambientes de Caatinga, sob diferentes níveis de conservação. A área de estudo corresponde a um remanescente de Caatinga situado em uma Unidade de Conservação denominada Monumento Natural Grota do Angico, no Alto Sertão sergipano. Foram instaladas 15 parcelas de $20 \times 2 \mathrm{~m}$ em cada área, sendo amostrados todos os indivíduos de espécies lenhosas (vivas), com diâmetro ao nível do solo (DNS) menor que $3 \mathrm{~cm}$ e altura total mínima de $15 \mathrm{~cm}$, sendo analisados os índices de diversidade de Shannon-Weaver (H') e de Equabilidade de Pielou (J'), de Similaridade de Jacard, e o padrão de distribuição espacial das espécies pelo Índice de Payandeh. Foi possível observar a formação de dois grupos bem definidos. A maior parte das espécies amostradas apresentou um padrão de distribuição espacial agregado. O processo de regeneração natural está em fase inicial na área de Caatinga estudada, expressando o seu potencial de resiliência.

Palavras-chave: Semiárido, Floresta tropical seca, Dinâmica florestal.

\begin{abstract}
The knowledge of the natural regeneration in Caatinga is essential to understand the important ecological process, especially the dynamics, considering its climatic conditions associated. This work was carried out with the goal to comprehend the natural regeneration, investigating the resilience of three Caatinga environments under different conservation levels. The study area is a Caatinga remnant situated in a Conservation Unit called Natural Monument Grota do Angico, in Sergipe's Alto Sertão. Three areas were selected for this study: Primitive Zone, Extensive Use Zone and Recovery Zone. There were installed 15 plots $(2$ x $20 \mathrm{~m})$ in each area, being sampled all individuals of woody species (live) with diameter at soil level less than $3 \mathrm{~cm}$ and minimum total height of $15 \mathrm{~cm}$, being analyzed the Shannon-Weaver $(\mathrm{H}$ ') and Pielou Equability ( $\mathrm{J}$ ') diversity index, of Jacard's Similarity and the pattern of spatial distribution of species by the Pay andeh Index. Was possible to observe two group s well defined. Most of the species sampled presented an aggregated spatial distribution pattern. The natural regeneration process is in initial phase in the studied Caatinga area, expressing its resilience potential.
\end{abstract}

Keywords: Semiarid, Tropical dry forest, Forest dy namics.

Introdução
O conhecimento sobre a composição florística das espécies e do comportamento sucessional e ecológico, das comunidades existentes em uma área florestal, são de grande importância para tomar-se decisões sobre as atividades de recuperação e as ações de conservação nestes ambientes (Marangon et al. 2016). Neste sentido, é necessário compreender os processos ecofisiológicos da vegetação, analisando-se a adaptação e o estabelecimento das espécies, uma vez que não há uma constância no tempo e no espaço das condições dos habitats florestais sucessionais (Falcão et al. 2015).

Uma destas mudanças é consequência das atividades antrópicas que degradam os recursos florestais, como é visto claramente na vegetação de Caatinga, no Semiárido brasileiro (Mattos et al. 2015). Aliado a isto, ainda são poucos os estudos que tratam das interações entre este tipo de floresta e o meio ambiente (Marangon et al. 2016).

Devido ainda existir uma grande falta de informações a respeito da dinâmica dos ecossistemas do Bioma Caatinga, são necessários estudos que possam subsidiar propostas de manejo da sua vegetação, conciliando com a sustentabilidade da área, e, para isto, deve-se compreender a sua composição florística e a sua regeneração natural (Lucena et al. 2016), além de aspectos importantes da estrutura das comunidades vegetais que o compõem. Além disso, como a estrutura da Caatinga é muito complexa, há dificuldade até mesmo no estabelecimento de políticas de conservação da sua diversidade vegetal (Santana et al. 2016).

A Caatinga no estado de Sergipe está representada apenas por remanescentes florestais em decorrência dos grandes desmatamentos realizados neste Bioma, realçando a necessidade de políticas para manter a conservação desta vegetação (Ferraz et al. 2013). Uma alternativa para esta situação foi a criação da Unidade de Conservação Monumento Natural Grota do Angico, com o objetivo de salvaguardar a diversidade da flora e fauna de um significativo remanescente de Caatinga no Alto Sertão do estado de Sergipe, visando conservar e diminuir a degradação nesta área (Silva et al. 2016). Contudo, os autores afirmam que há uma deficiência na produção de dados e conhecimento sobre a vegetação desta UC. Isto se agrava ainda mais, quando se trata de estudos de regeneração da Caatinga.

Diante do exposto, este trabalho foi realizado com o objetivo de conhecer a regeneração natural, por meio da composição, diversidade e similaridade florística, e do padrão de distribuição espacial das espécies florestais da Caatinga, investigando-se a capacidade de resiliência de três ambientes (Zona Primitiva, Zona de Uso Extensivo e Zona de Recuperação), sob diferentes níveis de conservação, na Unidade de Conservação (UC) de Proteção Integral Monumento Natural Grota do Angico, no Semiárido do estado de Sergipe. 


\section{Material e métodos Área de estudo}

$\mathrm{O}$ estudo foi realizado em um remanescente de Caatinga dentro da Unidade de Conservação (UC) de Proteção Integral estadual, Monumento Natural Grota do Angico (MONA), localizado entre os municípios de Poço Redondo e Canindé de São Francisco, no Alto Sertão do estado de Sergipe, delimitado entre as coordenadas UTM X/Y: $637270,59 / 8935547,258 \mathrm{~m}$ e $649298,966 / 8925786,101 \mathrm{~m}$ Datum SIRGAS 2000. A UC possui uma área total de aproximadamente 2.138 ha, e está inserida na região semiárida, cuja precipitação anual é inferior a $700 \mathrm{~mm}$ e a temperatura média de varia de 24 a $26^{\circ} \mathrm{C}$, e o clima é do tipo BSh, pela classificação de Köppen (Alvares et al. 2014).

A vegetação do MONA Grota do Angico foi classificada por Ribeiro e Mello (2007) em duas fitofisionomias: uma vegetação fechada de florestas hipoxerófita caducifólia e uma vegetação aberta em estádio de regeneração, caracterizada por pastagens e áreas abandonadas com solos salinizados. Os solos da região são caracterizados pela presença de 2 tipos: Neossolo Litólico e Planossolo (Leite 1976).

Para a escolha das áreas de estudo considerou-se o Zoneamento Ambiental apresentado no Plano de Manejo do Monumento Natural Grota do Angico, o qual define o grau de interferência permitido para as diferentes regiões da UC. Neste plano, foram definidas oito diferentes zonas: Primitiva, Uso Extensivo, Uso Intensivo, Histórico/Cultural, Recuperação, Uso Especial, Uso Conflitante e Ocupação. Para a realização deste trabalho, foram selecionadas três áreas: a Zona Primitiva, que envolve os remanescentes mais conservados de Caatinga; a Zona de Uso Extensivo, considerada uma zona de nível médio de degradação; e, por fim, a Zona de Recuperação, uma área de Caatinga muito alterada, que deve ser futuramente recuperada para atingir um melhor estado de conservação (SEM ARH 2011).

Para a realização das análises da regeneração natural, foram instaladas 5 parcelas de $20 \times 2 \mathrm{~m}$ em cada um dos ambientes selecionados, totalizando 15 unidades amostrais e equivalendo a uma área total de $600 \mathrm{~m} 2$. As parcelas foram dispostas paralelamente à margem do Rio São Francisco (Figura 1), obedecendo-se à distância de $50 \mathrm{~m}$ uma da outra.

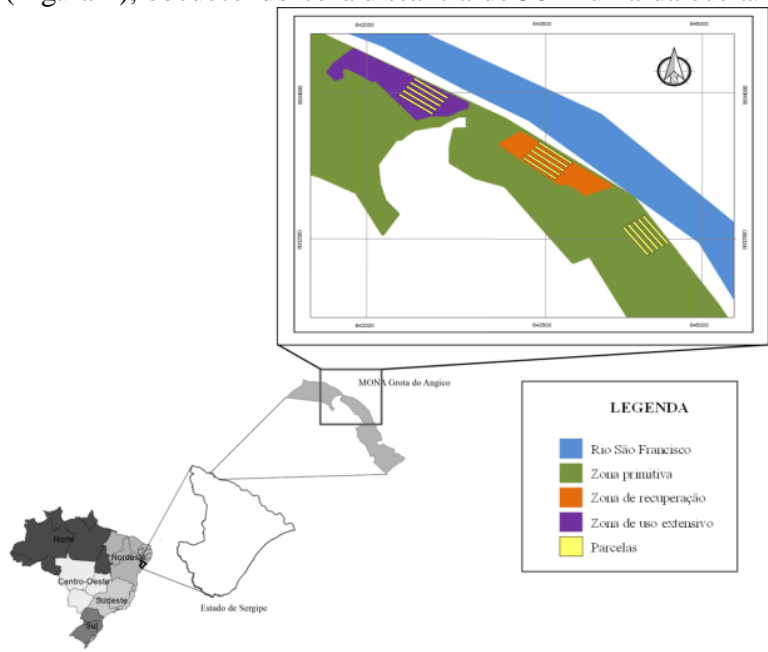

Figura 1. Disposição das parcelas instaladas no MONA Grota do Angico, Sergipe, 2012 para análise da regeneração natural.

Os levantamentos dos dados sobre o estrato regenerativo ocorreram por meio de duas avaliações, realizadas nos meses de janeiro e maio de 2012, sendo amostrados todos os indivíduos de espécies lenhosas (vivas), com diâmetro ao nível do solo (DNS) menor que $3 \mathrm{~cm}$ e altura mínima de 15 $\mathrm{cm}$, medidos, respectivamente, com o auxílio de um paquímetro analógico de plástico com precisão de $0,05 \mathrm{~mm}$, e uma escala métrica de madeira, com graduação em milímetros.

Também foram coletados materiais botânicos de todas as espécies encontradas nas unidades amostrais, que posteriormente foram devidamente herborizados, segundo as normas usuais sugeridas por Mori et al. (1989), os quais foram prensados, colocados em estufa e em seguida, identificados no Herbário da Universidade Federal de Sergip e (ASE), e as espécies foram classificadas de acordo com o Angiosperm Phy logeny Group (APG IV 2016).

\section{Diversidade florística}

Para avaliar a diversidade florística dos ambientes, foram empregados os índices de Diversidade de Shannon-Weaver (H') e de Equabilidade de Pielou (J'), conforme Brower \& Zar (1984). O índice de Diversidade de Shannon-Weaver é muito utilizado para quantificar a diversidade de espécies em estudos ecológicos, e depende basicamente da riqueza de espécies em determinada comunidade e da uniformidade com que os índivíduos são distribuídos entre as espécies (Begon et al. 1996). Quanto maior for o valor de $\mathrm{H}^{\prime}$, maior será a diversidade florística da comunidade em estudo, sendo este índice calculado pela seguinte equação:

$$
\mathrm{H}^{\prime}=\frac{\mathrm{N} * \ln (\mathrm{N})-\sum_{\mathrm{i}=1}^{\mathrm{S}} \mathrm{n}_{\mathrm{i}} * \ln \left(\mathrm{n}_{\mathrm{i}}\right)}{\mathrm{N}}
$$

Em que:

$\mathrm{H}^{\prime}=$ Índice de Diversidade de Shannon-Weaver;

$\mathrm{N}=$ número total de indivíduos amostrados; $\mathrm{n}_{\mathrm{i}}=$ número de indivíduos amostrados da i-ésima espécie; $\mathrm{S}$ = número de espécies amostradas; $\ln =$ logaritmo de base neperiana (e)

$O$ índice de Equabilidade de Pielou representa a proporção da diversidade de espécies encontradas na amostragem atual em relação à diversidade máxima que a comunidade poderá atingir (Brower; Zar 1984) e também, a uniformidade da repartição dos indivíduos entre as espécies (Odum 1986). Este índice pertence ao intervalo [0,1], onde 1 representa a máxima diversidade, ou seja, todas as espécies são igualmente abundantes. $\mathrm{O}$ valor da Equabilidade de Pielou é obtido pela expressão:

$$
\mathrm{J}^{\prime}=\frac{\mathrm{H}^{\prime}}{\ln (\mathrm{S})}
$$

Em que:

$\mathrm{J}$ '= Índice de Equabilidade de Pielou;

H'= Índice de Diversidade de Shannon-Weaver; $\mathrm{S}=$ número total de espécies.

\section{Similaridade florística}

O grau de similaridade florística entre os ambientes amostrados foi determinado por meio da comparação entre os mesmos, utilizando-se o Índice de Similaridade de Jaccard (Jaccard 1901). Este índice considera médias binárias com a convenção $1=$ presença e $0=$ ausência, sendo calculado pela expressão a seguir:

$$
S_{j}=\frac{a}{(a+b+c+d)}
$$

Em que:

$\mathrm{S}_{\mathrm{j}}=$ Coeficiente de Similaridade de Jaccard;

$\mathrm{a}=$ número de espécies comuns em todas as áreas;

$\mathrm{b}=$ número de espécies únicas da área $\mathrm{A}$;

$\mathrm{c}=$ número de espécies únicas da área $\mathrm{B}$

$\mathrm{d}=$ número de espécies únicas da área $\mathrm{C}$. 
Os dados de diversidade florística e padrão espacial das espécies foram analisados no Mata Nativa 2.10® (CIENTEC 2006), sendo os resultados da diversidade florística posteriormente submetidos à análise de variância (ANOVA) através do programa estatístico $\mathrm{R}$ ( $\mathrm{R}$ Development Core Team 2011). Para os dados de similaridade foi utilizado o software PAST v1.34 (Hammer et al. 2001).

\section{Resultados e discussão}

$\mathrm{Na}$ análise da composição florística, 17 táxons foram identificados ao nível de espécie, 2 ao nível de gênero e 1 ficou indeterminado. Ao todo, foram amostrados 333 indivíduos pertencentes a 12 famílias botânicas, em 19 gêneros, contendo 20 espécies, sendo duas não identificadas (Tabela 1). Tais resultados mostraram-se superiores aos obtidos por Fabricante e Andrade (2007), em um levantamento da regeneração natural realizado em uma área de $4.000 \mathrm{~m}^{2}$ no Semiárido paraibano, no qual encontraram 1.129 indivíduos, pertencentes a 7 famílias, distribuídas em 15 espécies.

Tabela 1. Relação de espécies encontradas no est rato regenerativo do MONA Grota do Angico, Sergipe, 2012. N1 = número de indivíduos amostrados no primeiro levantamento, $\mathrm{N} 2=$ número de indivíduos amostrados no segundo levantamento, $\mathrm{ZP}=$ Zona Primitiva, $\mathrm{ZR}=$ Zona de Recuperação e ZE = Zona de Uso Extensivo.

\begin{tabular}{|c|c|c|c|c|c|}
\hline \multirow[b]{2}{*}{ Familia/ Espécies } & \multicolumn{2}{|c|}{ Levantamentos } & \multicolumn{3}{|c|}{ Zonas } \\
\hline & N1 & N2 & $\mathrm{ZP}$ & $\mathrm{ZR}$ & $\overline{\mathrm{ZE}}$ \\
\hline $\begin{array}{l}\text { Anacardiaceae } \\
\end{array}$ & & & & & \\
\hline Myracrodruon urundeuva Allemão & 1 & 1 & & & $\mathrm{x}$ \\
\hline Schinopsis brasiliensis Engl. & 1 & 0 & & & $\mathrm{x}$ \\
\hline Apocynaceae & & & & & \\
\hline $\begin{array}{c}\text { Aspidosperma pyrifolium Mart. \& Zucc. } \\
\text { Boraginaceae }\end{array}$ & 6 & 12 & $\mathrm{x}$ & $\mathrm{x}$ & $\mathrm{x}$ \\
\hline Varronia globosa (Jacq.) Kunth & 0 & 3 & $\mathrm{x}$ & $\mathrm{x}$ & \\
\hline Burseraceae & & & & & \\
\hline $\begin{array}{c}\text { Commiphora leptophloeos (Mart.) J.B. Gillett } \\
\text { Capparaceae }\end{array}$ & 34 & 68 & $\mathrm{x}$ & $\mathrm{x}$ & $\mathrm{x}$ \\
\hline $\begin{array}{l}\text { Cynophalla flexuosa }(\mathrm{L} .) \mathrm{L} \text {. } \\
\text { Celastraceae }\end{array}$ & 3 & 3 & & & $\mathrm{x}$ \\
\hline $\begin{array}{l}\text { Maytenus rigida Mart. } \\
\text { Erythroxylaceae }\end{array}$ & 9 & 11 & & & $\mathrm{x}$ \\
\hline Erythroxylum sp. & 0 & 3 & $\mathrm{x}$ & $\mathrm{x}$ & \\
\hline $\begin{array}{c}\text { Jatropha mollissima (Pohl) Baill. } \\
\text { Fabaceae }\end{array}$ & 23 & 23 & $\mathrm{x}$ & $\mathrm{x}$ & \\
\hline Anadenanthera colubrina (Vell.) Brenan & 7 & 15 & & $\mathrm{x}$ & \\
\hline Bauhinia cheilantha (Bong.) Steud. & 18 & 23 & $\mathrm{x}$ & & $\mathrm{x}$ \\
\hline Libidibia ferrea (Mart. ex Tul.) L.P.Queiroz & 1 & 1 & $\mathrm{x}$ & & \\
\hline Mimosa tenuiflora (Willd.) Poir. & 1 & 0 & & & $\mathrm{x}$ \\
\hline Mimosa sp. & 0 & 1 & & & $\mathrm{x}$ \\
\hline Piptadenia stipulacea (Benth.) Ducke & 0 & 1 & & $\mathrm{x}$ & \\
\hline $\begin{array}{c}\text { Poincianella pyramidalis (Tul.) L.P.Queiroz } \\
\text { Rhamnaceae }\end{array}$ & 22 & 26 & $\mathrm{x}$ & $\mathrm{x}$ & $\mathrm{x}$ \\
\hline Ziziphus joazeiro Mart. & 1 & 3 & $\mathrm{x}$ & $\mathrm{x}$ & \\
\hline $\begin{array}{l}\text { Sapotaceae } \\
\text { Sideroxylon obtusifolium (Roem. \& Schult.) T.D. } \\
\text { Penn. }\end{array}$ & 3 & 4 & $\mathrm{x}$ & & $\mathrm{x}$ \\
\hline $\begin{array}{l}\text { Solanaceae } \\
\end{array}$ & & & & & \\
\hline $\begin{array}{l}\text { Capsicum caatingae Sendtn. } \\
\text { Indeterminada }\end{array}$ & 1 & 2 & $\mathrm{x}$ & $\mathrm{x}$ & \\
\hline $\begin{array}{l}\text { Sp. I } \\
\text { Sp. II }\end{array}$ & 1 & 1 & & & $\mathrm{x}$ \\
\hline
\end{tabular}

Analisando-se a totalidade das áreas estudadas, a família com maior riqueza de espécies no estrato regenerativo foi Fabaceae (7), representando 31,25 e 33,3\% das espécies encontradas na primeira e segunda avaliação, respectivamente.

Embora não seja a família mais rica em espécies, Burseraceae constituiu uma alta abundância (102 indivíduos), e junto à Fabaceae (116 indivíduos), representaram 65,46\% dos espécimes levantados em todo o estudo. Este resultado tem grande influência da presença de Commiphora leptophloeos, Poincianella pyramidalis e Bauhinia cheilantha, pois foram as espécies com maior quantidade de indivíduos na área de estudo. A espécie mais abundante foi Commiphora leptophloeos, com 102 indivíduos, respondendo por $33,63 \%$ do total amostrado. Em estudos realizados em outros fragmentos de Caatinga, também na UC MONA
Angico, por Ferraz et al. (2013) e Silva et al. (2016), os autores identificaram que Poincianella pyramidalis foi a mais representativa, seguida de Jatropha mollissima e Bauhinia cheilantha.

A ocorrência da espécie de maior população (Commiphora leptophloeos) foi registrada no ambiente em melhor estado de conservação (Zona Primitiva), correspondendo a 94\% dos indivíduos deste táxon. Este resultado pode estar relacionado ao fato de que a referida espécie raramente ocorre em áreas de forte antropização, sendo geralmente encontrada em ambientes mais protegidos, ou bem conservados do Bioma (Andrade et al. 2005).

Dos 20 táxons encontrados, neste estudo, os menores valores de abundância foram registrados para 6 espécies, as quais apresentaram apenas um indivíduo em toda a amostragem. Fazem parte desse grupo Schinopsis brasiliensis, Mimosa tenuiflora, Mimosa sp., Piptadenia stipulacea e Sp.I e II (Indeterminada). Os táxons que apresentaram ocorrência nas três zonas estudadas foram: Aspidosperma pyrifolium, Commiphora leptophloeos e Poincianella pyramidalis, demonstrando melhor adaptação dessas espécies aos ambientes estudados, de melhor conservação ou em condição de antropização ambiental.

Na Zona Primitiva, foi registrada a ocorrência de 9 famílias, 10 gêneros e 10 espécies e na Zona de Recuperação (ambiente de maior antropização), foi observada a presença de 8 famílias, 11 gêneros e 11 espécies, correspondendo a um gênero e uma a mais do que a Zona Primitiva. A Zona de Uso Extensivo (ambiente de nível médio de degradação) apresentou valores superiores às demais, contando com 13 espécies (sendo 2 indeterminadas), distribuídas 13 gêneros e em 9 famílias (considerando apenas as identificadas), ocorrendo uma exclusividade de 8 táxons apenas neste ambiente. Isto pode evidenciar uma maior adaptação das espécies a esse ambiente, caracterizado segundo o plano de manejo como uma zona de grau de degradação intermediário entre as demais.

Baseando-se no trabalho realizado por Pereira et al. (2001), em uma outra região da Caatinga, esperar-se-ia que a riqueza de espécies no ambiente mais conservado fosse maior e com menor abundância de indivíduos, porém ocorreu justamente o contrário, pois esse ambiente se apresentou menos diverso e mais populoso, em relação ao estrato regenerante.

Dentre os 333 indivíduos juvenis identificados e medidos, no presente estudo, 132 foram encontrados na primeira avaliação (janeiro de 2012) e 201 na segunda (maio de 2012), indicando o favorecimento da regeneração do ambiente nesta última avaliação, que corresponde a um mês da estação chuvosa da região. A razão para esse acréscimo pode estar relacionada aos eventos de frutificação, dispersão e germinação de sementes de algumas espécies, que são comportamentos fenológicos sujeitos direta ou indiretamente aos determinantes climáticos.

Em termos gerais, uma maior quantidade de espécies lenhosas no estádio de regeneração foi observada no ambiente mais conservado (Zona Primitiva), em especial na segunda avaliação (111 indivíduos). Enquanto isso, a menor quantidade foi registrada na Zona de Recuperação, durante a primeira avaliação (30 indivíduos). Esse quadro sugere um ap arente favorecimento dos processos de restabelecimento vegetal em ecossistemas menos degradados.

Porém, deve-se levar em consideração que o fator que mais influenciou nesse resultado foi a presença de Commiphora leptophloeos, a qual apresentou uma grande abundância nesse ambiente, sendo responsável por 53\% dos indivíduos amostrados na referida área. A ocorrência significante desse táxon pode justificada por diversos aspectos, como por exemplo, as suas próprias características 
fenológicas, não sendo determinada apenas pelas condições ambientais do local onde predomina. Avaliando-se desta forma, talvez o nível de conservação não represente um fator determinante da elevada abundância na Zona Primitiva. Por outro lado, Cabral et al. (2013) analisando diferentes estádios sucessionais de Caatinga, em Santa Terezinha, Paraíba, identificaram que Mimosa tenuiflora foi o táxon dominante no estádio inicial, enquanto que Poincianella pyramidalis se destacou no estádio mais tardio da sucessão, diferentemente, dos resultados encontrado no presente estudo.

\section{Diversidade florística}

Pelos Índices de Diversidade de Shannon-Weaver ( $\left.\mathrm{H}^{\prime}\right)$ obteve-se 2,13 nats.indivíduo ${ }^{-1}$ no primeiro levantamento e 2,16 nats.indivíduo ${ }^{-1}$ no segundo (Tabela 2 ), sendo inferior ao observado por Lima et al. (2011) 3,26 nats.indivíduo ${ }^{-1}$, em trabalho realizado na Reserva Natural Serra das Almas, Ceará, e por Cordeiro et al. (2017), que obtiveram um valor de 3,196 nats.indivíduo ${ }^{-1}$ em um fragmento da Floresta Estacional Decidual, PB. Mas os resultados obtidos foram superiores aos encontrado por Barbosa et al. (2012), em uma área de Caatinga do sertão pernambucano, correspondente a 2,05 nats.indivíduo ${ }^{-1}$. Os Índices de Equabilidade de Pielou (J') nos dois levantamentos também superaram o valor encontrado por esses autores $(0,57)$, sendo 0,77 na primeira avaliação e 0,75 na segunda (Tabela 2). Esses valores também foram superiores aos de Silva et al. (2016), que observaram um índice de Diversidade de Shannon-Weaver de 1,72 e de Equabilidade de Pielou de 0,5, em um fragmento da Caatinga também situado no MONA Angico.

Tabela 2. Índices de diversidade de Shannon-Weaver (H') e Equabilidade de Pielou (J') de três ambientes estudados, nos meses de janeiro e maio de 2012. MONA Grota do Angico, Sergipe, 2012.

\begin{tabular}{lcccc}
\hline \multirow{2}{*}{ Zona/ Parcela } & \multicolumn{2}{c}{ Janeiro } & \multicolumn{2}{c}{ Maio } \\
\cline { 2 - 5 } & H' & J' & H' & J' \\
\hline Zona Primitiva & 1,21 & 0,75 & 1,35 & 0,59 \\
Zona de Recuperação & 1,80 & 0,87 & 1,84 & 0,84 \\
Zona de Uso Extensivo & 1,99 & 0,83 & 1,75 & 0,76 \\
\hline *** Total & $\mathbf{2 , 1 3}$ & $\mathbf{0 , 7 7}$ & $\mathbf{2 , 1 6}$ & $\mathbf{0 , 7 5}$ \\
\hline
\end{tabular}

Comparando-se a diversidade de Shannon-Weaver por Zona, nota-se que na Zona de Uso Extensivo foi superior aos demais ambientes na primeira avaliação (1,99 nats.indivíduo ${ }^{1}$ ) e a Zona de Recuperação na segunda (1,84 nats.indivíduo $\left.{ }^{1}\right)$. Embora aparentemente tenham ocorrido variações, a análise de variância (ANOVA) apontou que esses valores não diferem entre si, determinando uma homogeneidade entre a diversidade de espécies que ocorrem nas três zonas, tanto na primeira $(\mathrm{F}=0,1699 ; \mathrm{df}=14 ; p=0,8457 ; \mathrm{N}=15)$, quanto na segunda avaliação $(\mathrm{F}=0,1997 ; \mathrm{df}=14 ; p=0,8217 ; \mathrm{N}=15)$.

O Índice de Equabilidade de Pielou (J') indicou, a partir dos valores obtidos nas duas avaliações $\left(\mathrm{J}^{\prime}=0,77\right.$ e 0,75$)$, que a diversidade entre os três ambientes estudados encontra-se em alta uniformidade, nas proporções do número de indivíduos/número de espécies dentro da comunidade vegetal.

Comparando-se os resultados das Zonas analisadas com outros fragmentos em diferentes estádios de conservação, o valor do Î́ndice de Shannon-Weaver, no presente estudo, foi menor e o de Pielou foi maior que o observado por Fernandes et al. (2017), 1,90 nats. ind ${ }^{-1}$ e 0,76, respectivamente, em uma área de desertificação em Gilbués-PI. O mesmo foi visto analisando-se a área preservada e a área em recuperação, com os resultados obtidos por Ferreira et al. (2016), com exceção apenas ao Índice de Pielou, no mês de maio para a Zona Primitiva, e por Ferraz et al. (2014) para o índice de ShannonWeaver, avaliando-se duas áreas (uma com cerca de 25 anos em recuperação, e outra com mais de 55 anos de conservação), com diferença também quanto ao Índice de Pielou para a mesma Zona, no mesmo período de avaliação.

Comparando-se os ambientes estudados, foi possível observar que as três zonas possuíam uma alta uniformidade entre a diversidade das suas parcelas. Entretanto, a Zona Primitiva, na segunda avaliação, apresentou o valor mais distante da uniformidade máxima $(0,59)$. A homogeneidade observada quanto à diversidade florística dos três ambientes pode ter sofrido influência de vários fatores, dentre eles: (1) o fato das três zonas possuírem o mesmo limite de distância (50 m) em relação ao Rio São Francisco; (2) a proximidade entre os ambientes; (3) o número de unidades amostrais instaladas para este estudo, sendo necessário o aumento do esforço amostral, a fim de confirmar ou rejeitar tal característica.

\section{Similaridade florística}

A análise do "diagrama de distância de ligação" ou "dendrograma de similaridade florística" indica a formação de dois grupos bem definidos, sendo o primeiro grupo formado pelas parcelas localizadas na Zona Primitiva, caracterizada por uma vegetação em bom estado de conservação, e pela Zona de Recuperação, ambiente de maior grau de antropização deste estudo (Figura 2). Este grup o pode ser dividido em dois subgrupos: um representado por três parcelas pertencentes à Zona de Recuperação mais próximas do rio $(50-150 \mathrm{~m})$ e o outro pelas demais parcelas, pertencentes tanto à Zona de Recuperação quanto à Zona Primitiva. O segundo grupo, foi composto pelas parcelas localizadas no interior da Zona de Uso Extensivo, uma área em estado de conservação intermediário entre as demais zonas.

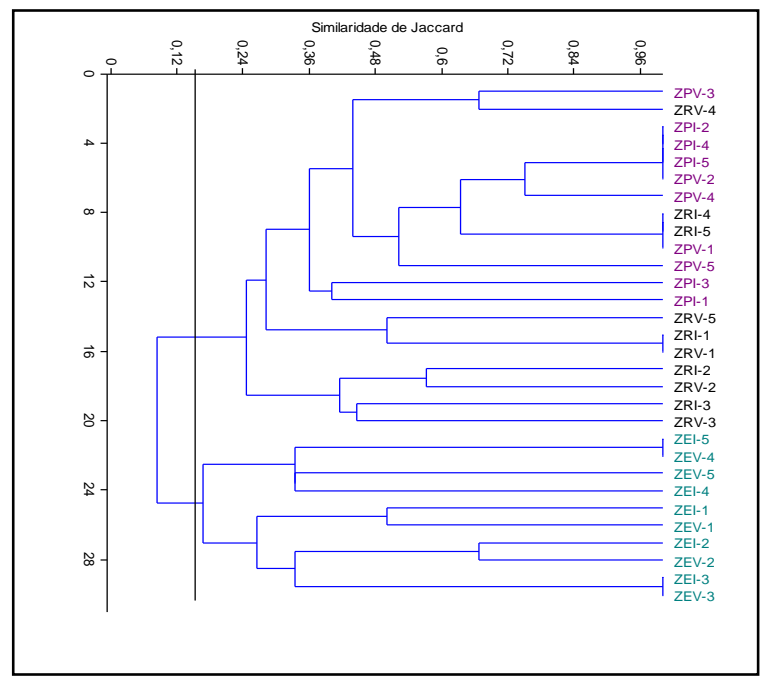

Figura 2. Dendrograma de similaridade florística entre Zona primitiva, Zona de recuperação e Zona de uso extensivo, no MONA Grota do Angico, Sergipe, 2012. ZP = Zona primitiva; ZR = Zona de recuperação; $\mathrm{ZE}=\mathrm{Z}$ ona de uso extensivo; $\mathrm{V}=$ verão; $\mathrm{I}=$ inverno; $1,2,3,4$ e $5=\mathrm{n}^{\circ}$ da parcela.

Os dados evidenciados no dendrograma acima, são, no geral, um resultado da diferença florística apresentada pelo estrato regenerante da Zona de Uso Extensivo. Esta diferenciação florística entre ambientes sob diferentes estágios de perturbação também foi constatado por Holanda et al. (2015), considerando-se tanto para os indivíduos adultos quanto para a regeneração natural, como por Ferreira et al. 
(2016) comparando-se uma área de Caatinga preservada com uma área em regeneração.

Nesta região de estudo, foram encontradas 8 espécies diferentes das que ocorreram nas demais zonas, e isto levou à formação de um grupo isolado. $\mathrm{O}$ arranjo desses grupos e subgrupos pode ter influência, dentre outros fatores, da localização geográfica e da intensidade de uso das áreas pelas comunidades humanas locais.

Para explicar melhor essa hipótese, é essencial relatar que as zonas Primitiva e de Recuperação são vizinhas e situam-se mais próximas aos povoados e assentamentos rurais da região, enquanto que a Zona de Uso Extensivo localiza-se em um área mais distante e com difícil acesso. Desta forma, o afastamento tanto de pessoas, como de animais domésticos, poderia ter aumentado as chances de perpetuação de um maior número de espécies nessa última zona.

\section{Conclusão}

As áreas de Caatinga estudadas no MONA Grota do Angico mostraram, em seus diferentes níveis de conservação, a sua capacidade de regeneração, indicando que a atividade antrópica não afetou o potencial do processo regenerativo dos ambientes avaliados, o qual já se encontra em fase inicial. Isto pode ser constatado análises florísticas, que indicaram que as alterações no estrato juvenil não foram diretamente proporcionais ao grau de degradação.

Foi constatada uma uniformidade entre os valores de diversidade dos ambientes, fornecida pelos cálculos dos Índices de Diversidade de Shannon-Weaver e Equabilidade de Pielou, bem como uma distinção na similaridade florística entre os ambientes selecionados e um agrupamento das espécies ao longo do espaço estudado.

Este estudo permitiu gerar informações que podem caracterizar inicialmente o potencial de reconstrução da vegetação, possibilitando, junto a um monitoramento contínuo destes ambientes, definir estratégias para o uso dos ambientes, por meio de manejo sustentável e, ainda, definir estratégias para conservação da biodiversidade da flora, não somente do MONA Grota do Angico, como para outros remanescentes do Bioma Caatinga.

\section{Referências}

Alvares CA, Stape JL, Sentelhas PC, Gonçalves JL de M, Sparovek G (2014) Köppen's climate classification map for Brazil. Meteorologische Zeitschrift, 22(6):711-728. doi: 10.1127/0941-2948/2013/0507.

Andrade LA, Pereira IM ,Leite UT, Barbosa MRV (2005) Análise da cobertura de duas fitofisionomias de caatinga, com diferentes históricos de uso, no município de São João do Cariri, estado da Paraíba. Cerne, 11(3):253-262.

APG IV (2016) An update of the Angiosperm Phylogeny Group classification for the orders and families of flowering plants: APG IV. Botanical Journal of the Linnean Society. London 181: 1-20.

Barbosa MD, Marangon LC, Feliciano ALP, Freire FJ, Duarte GMT (2012) Florística e fitossociologia de espécies arbóreas e arbustivas em uma área de Caatinga em Arcoverde, PE, Brasil. Revista Árvore, 36(5):851-858. doi: http://dx.doi.org/10.1590/S0100-67622012000500007.

Begon M, Harper JL, Townsend CR (1996) Ecology: individuals, populations and communities. $3^{\text {th }}$ Edition. Oxford: Blackwell, 1068p.
Brower JE, Zarr JH (1984) Field \& laboratory methods for general ecology. $2^{\text {a }}$ Edition. Iowa: Wm. C. Brown Company, 226p.

Cabral GAL, Sampaio EVSB, Almeida-Cortez JC (2013) Estrutura Espacial e Biomassa da Parte Aérea em Diferentes Estádios Sucessionais de Caatinga, em Santa Terezinha, Paraíba. Revista Brasileira de Geografia Física, 6(3):566-574

CIENTEC (2006) Mata Nativa 2: Sistema para análise fitossociológica e elaboração de planos de manejo de florestas nativas. Viçosa. 295p.

Cordeiro JMP, Bartolomeu IS, Feliz LP (2017) Florística e fitossociologia em Floresta Estacional Decidual na Paraíba, nordeste do Brasil. Gaia Scientia, 11(1):1-16. doi: https://doi.org/10.22478/ufpb.1981-

$1268.2017 \mathrm{v} 11 \mathrm{n} 1.33245$.

Fabricante JR, Andrade LA (2007) Análise estrutural de um remanescente de caatinga no Seridó Paraibano. Oecologia Brasiliensis, 11(3):341-349.

Falcão HM, Medeiros CD, Silva BLR, Sampaio EVSB, Almeida-Cortez JS, Santos MG (2015) Phenotypic plasticity and ecophysiological strategies in a tropical dry forest chronosequence: A study case with Poincianella pyramidalis. Forest Ecology and Management, 340: 62-69. doi: 10.1016/j.foreco.2014.12.029.

Fernandes MM, Oliveira TM, Fernandes MRM (2017) Regeneração natural de um fragmento florestal de caatinga na região semi-árida do Piauí. Scientia Plena, 13(2):7p. doi: https://doi.org/10.14808/sci.plena.2017.021701.

Ferraz RC, Mello AA, Ferreira RA, Prata APN (2013) Levantamento fitossociológico em área de caatinga no Monumento Natural Grota Do Angico, Sergipe, Brasil. Revista Caatinga, 26(3):89-98.

Ferraz JSF, Ferreira RLC, Silva JAA, Meunier MJ, Santos M VF (2014) Estrutura do componente arbustivo-arbóreo da vegetação em duas áreas de caatinga, no município de Floresta, Pernambuco. Revista Árvore, 38(6):1055-1064. doi: http://dx.doi.org/10.1590/S0100 67622014000600010 .

Ferreira RLC, Silva SO, Silva JAA, Lira MA, Alves Júnior FT, Nascimento LM (2016) Richness and diversity of Caatinga areas in diferente successional stages in northeastern Brazil. Scientia Forestalis, 44(112):799-810. doi: dx.doi.org/10.18671/scifor.v44n112.02.

Hammer O, Harper DAT, Ryan PD (2001) Past: paleontological statistics software package for education and data analy sis. Paleontologia Electronica, 4:1-9.

Holanda AC, Lima FTD, Silva BM, Dourado RG, Alves AR (2015) Estrutura da vegetação em remanescentes de caatinga com diferentes históricos de perturbação em Cajazeirinhas (PB). Revista Caatinga, 28(4):142-150. doi: http://dx.doi.org/10.1590/1983-21252015v28n416rc.

Jaccard P (1901) Etude comp arative de la distribution florare dans une portion des Alpes et du Jura. Bulletin Société Vaudoise dês Sciences Naturelles, 37:547-579.

Leite JAN, Araújo LVC, Arriel EF, Chaves LFC, Nóbrega AMF (2015) Análise quantitativa da vegetação lenhosa da 
Caatinga em Teixeira, PB. Pesquisa Florestal Brasileira, 35(82):89-100. https://doi.org/10.4336/2015.pfb.35.82.584.

doi:

Leite LW (1976) Zoneamento ecológico-florestal do estado de Sergipe. Aracaju: Sudene/Condese. 107p.

Lima JR, Sampaio EVSB, Rodal MJN, Araújo FS (2011) Phy siognomy and structure of a seasonal deciduous forest on the Ibiapaba plateau, Ceará, Brazil. Rodriguésia, 62(2):379-389. doi: http://dx.doi.org/10.1590/2175 7860201162212 .

Lucena MS, Silva JA, Alves AR (2016) Regeneração natural do estrato arbustivo-arbóreo em área de Caatinga na Estação Ecológica do Seridó - RN, Brasil. Biotemas, 29 (2):17-31, 2016. doi: https://doi.org/10.5007/21757925.2016v29n2p 17.

Marangon GP, Felke RM,Zimmermann APL, Ferreira RLC, Silva JAA (2016) Análise de agrupamento de espécies lenhosas da Caatinga no Estado do Pernambuco. Pesquisa Florestal Brasileira, 36(88):347-353. doi: https://doi.org/10.4336/2016.pfb.36.88.1030.

Mattos PV, Braz EM, Domene VD, Sampaio EVSB, Gasson P, Pareyn FGC, Alvarez IA, Baracat A, Araújo EL (2015) Climate-tree growth relationships of Mimosa tenuiflora in seasonally dry tropical forest, brazil. Cerne, 21(1):141-149. doi: http://dx.doi.org/10.1590/01047760201521011460.

Mori AS, Silva LAM, Lisboam G, Corandin L (1989) Manual de manejo do herbário fanerogâmico. Ilhéus: Centro de Pesquisa de Cacau. 43p.

Odum EP (1986) Ecologia. Rio de Janeiro: Guanabara, 434p. Pereira IM, Andrade LA, Costa JRM, Dias JM (2001) Regeneração natural em um remanescente de Caatinga sob diferentes níveis de perturbação, no Agreste Paraibano. Acta Botânica Brasílica, 15(3):413-426.

R Development Core Team (2011) $R$ : A language and environment for statistical computing. R Foundation for Statistical Computing, Vienna. Disponível em: <http://www.R-project.orgS.>. Acesso em 20 de julho de 2011.

Ribeiro AS, Mello AA (2007) Diagnóstico da biota. In: Ribeiro AS (Coord.) Estudos para criação do Monumento Natural Grota do Angico. Sergipe: Governo de Sergipe, Secretaria de Estado do Meio Ambiente e dos Recursos Hídricos. 51p.

Santana JAS, Santana Júnior JAS, Barreto WS, Ferreira ATS (2016) Estrutura e distribuição espacial da vegetação da Caatinga na Estação Ecológica do Seridó, RN. Pesquisa Florestal Brasileira, 36(88):355-361. doi: https://doi.org/10.4336/2016.pfb.36.88.1002.

SEMARH (2011) Secretaria de Estado do Meio Ambiente e dos Recursos Hídricos de Sergipe. Plano de Manejo do Monumento Natural Grota do Angico. 614f.

Silva ACC, Prata APN, Mello AA (2016) Florística, fitossociologia e caracterização sucessional em um remanescente de Caatinga em Sergipe. Gaia Scientia, $10(4): 1-14$ 